\title{
AN ITERATIVE APPROACH TO A CONSTRAINED LEAST SQUARES PROBLEM
}

\author{
SIMEON REICH AND HONG-KUN XU
}

Received 3 December 2001

A constrained least squares problem in a Hilbert space $H$ is considered. The standard Tikhonov regularization method is used. In the case where the set of the constraints is the nonempty intersection of a finite collection of closed convex subsets of $H$, an iterative algorithm is designed. The resulting sequence is shown to converge strongly to the unique solution of the regularized problem. The net of the solutions to the regularized problems strongly converges to the minimum norm solution of the least squares problem if its solution set is nonempty.

\section{Introduction}

Consider the following constrained least squares problem in a Hilbert space $H$ :

$$
\min _{x \in C} f(x):=\frac{1}{2}\|A x-b\|^{2}
$$

where $C$ is a nonempty closed convex subset of $H, A: H \rightarrow H$ is a bounded linear operator, and $b$ is an element of $H$.

Let $S$ denote the solution set of (1.1). It is known that $S$ is closed and convex, but it may well be empty.

Since problem (1.1) may be ill-posed, a regularization process is necessary in order to find a solution of (1.1) (if any). We will apply the standard Tikhonov regularization to (1.1); that is, we consider the following regularized problem $(\varepsilon>0)$ :

$$
\min _{x \in C} f_{\varepsilon}(x):=\frac{1}{2}\|A x-b\|^{2}+\frac{1}{2} \varepsilon\|x\|^{2} .
$$


(The reader is referred to [6] by Engl et al. for regularization methods for inverse and ill-posed problems. See also Tikhonov and Arsenin [12] and Björck [3].)

Since $f_{\varepsilon}$ is continuous, convex, and coercive (i.e., $\|x\| \rightarrow \infty \Rightarrow f_{\varepsilon}(x) \rightarrow \infty$ ), (1.2) has a unique solution which we denote by $x_{\varepsilon}$.

In this paper, we first discuss the asymptotic behavior of $\left\{x_{\varepsilon}\right\}$ as $\varepsilon \downarrow 0$ and then design an iterative algorithm which produces a sequence converging strongly to the unique solution $x_{\varepsilon}$ of (1.2) in the case where $C$ is the nonempty intersection of a finite collection of closed convex subsets of $H$. Moreover, if $S$ is nonempty, then the net $\left\{x_{\varepsilon}\right\}$ of the approximate solutions converges strongly to the minimum norm solution of (1.1).

\section{Behavior of $\left\{x_{\varepsilon}\right\}$}

Let $C, f, f_{\varepsilon},\left\{x_{\varepsilon}\right\}$, and $S$ be given as in the introduction. It is the objective of this section to study the behavior of the net $\left\{x_{\varepsilon}\right\}$ of the solutions of the regularized problem (1.2). The next result shows that as long as the solution set $S$ of (1.1) is nonempty, $\left\{x_{\varepsilon}\right\}$ behaves well and approaches the minimum norm solution of (1.1).

TheOREm 2.1. The behavior of $\left\{x_{\varepsilon}\right\}$ is as follows:

(i) $S \neq \varnothing$ if and only if $\left\{x_{\varepsilon}\right\}$ is bounded as $\varepsilon \downarrow 0$. In this case, $\left\{x_{\varepsilon}\right\}$ converges strongly as $\varepsilon \downarrow 0$ to the minimum norm solution of (1.1);

(ii) $S=\varnothing$ if and only if $\lim _{\varepsilon \downarrow 0}\left\|x_{\varepsilon}\right\|=\infty$.

Proof. To prove (i), first assume that $S \neq \varnothing$. Since $S$ is closed and convex, there is a unique element in $S$, denoted by $S^{\circ}$, such that

$$
\left\|S^{\circ}\right\|=\min \{\|v\|: v \in S\}
$$

Since $f_{\varepsilon}\left(x_{\varepsilon}\right) \leq f_{\varepsilon}\left(S^{\circ}\right)$, we see that

$$
\varepsilon\left(\left\|x_{\varepsilon}\right\|^{2}-\left\|S^{\circ}\right\|^{2}\right) \leq\left\|A S^{\circ}-b\right\|^{2}-\left\|A x_{\varepsilon}-b\right\|^{2} .
$$

But $f\left(S^{\circ}\right) \leq f\left(x_{\varepsilon}\right)$; that is, $\left\|A S^{\circ}-b\right\|^{2} \leq\left\|A x_{\varepsilon}-b\right\|^{2}$, it follows from (2.2) that

$$
\left\|x_{\varepsilon}\right\| \leq\left\|S^{\circ}\right\|
$$

Hence, $\left\{x_{\varepsilon}\right\}$ is bounded.

Next, assume that $\left\{x_{\varepsilon}\right\}$ is bounded as $\varepsilon \downarrow 0$. Assume that $\varepsilon_{n} \downarrow 0$ and $x_{\varepsilon_{n}} \rightarrow \bar{x}$ weakly. Clearly, $\bar{x} \in C$. 
We claim that $\bar{x} \in S$. To see this, we note that the weak lower semicontinuity of $f$ implies that for any $x \in C$,

$$
\begin{aligned}
f(\bar{x}) & \leq \liminf _{n \rightarrow \infty} f\left(x_{\varepsilon_{n}}\right) \leq \limsup _{\varepsilon \downarrow 0} f\left(x_{\varepsilon}\right)=\limsup _{\varepsilon \downarrow 0} f_{\mathcal{\varepsilon}}\left(x_{\varepsilon}\right) \\
& \leq \limsup _{\varepsilon \downarrow 0} f_{\varepsilon}(x)=f(x) .
\end{aligned}
$$

This shows that $\bar{x} \in S$.

Next, we prove, assuming that $S \neq \varnothing$, that $\left\{x_{\varepsilon}\right\}$ converges strongly as $\varepsilon \downarrow 0$ to $S^{\circ}$, the minimum norm solution of (1.1). Let $v$ be any weak limit point of $\left\{x_{\varepsilon}\right\}$ when $\varepsilon \downarrow 0$. Then, we have a sequence $\varepsilon_{n} \downarrow 0$ such that $x_{\varepsilon_{n}} \rightarrow v$ weakly. Since the norm of $H$ is weakly lower semicontinuous, it follows from (2.3) that

$$
\|v\| \leq\left\|S^{\circ}\right\|
$$

Due to the uniqueness of the minimum norm element, this implies that $v=S^{\circ}$, which in turn implies that $S^{\circ}$ is the only weak limit point of $\left\{x_{\varepsilon}\right\}$. Therefore, again, due to the weak lower semicontinuity of the norm and (2.3), we have

$$
w-\lim _{\varepsilon \downarrow 0} x_{\varepsilon}=S^{\circ}, \quad \lim _{\varepsilon \downarrow 0}\left\|x_{\varepsilon}\right\|=\left\|S^{\circ}\right\|
$$

This implies that $s-\lim _{\varepsilon \downarrow 0} x_{\varepsilon}=S^{\circ}$.

In order to prove (ii), we first note that if $S \neq \varnothing$, then by (i) $\left\{x_{\varepsilon}\right\}$ is bounded. So, it remains to show that $S=\varnothing$ implies

$$
\lim _{\varepsilon \downarrow 0}\left\|x_{\varepsilon}\right\|=\infty
$$

Suppose on the contrary that (2.7) does not hold. Then we can find a sequence $\left\{\varepsilon_{n}\right\} \downarrow 0$ and some $\tilde{x} \in C$ such that

$$
x_{\varepsilon_{n}} \longrightarrow \tilde{x} \quad \text { weakly. }
$$

Repeating the first half of the proof of part (i), we obtain that

$$
f(\tilde{x}) \leq f(x), \quad x \in C
$$

That is, $\tilde{x} \in S$ and $S$ is nonempty. The contradiction we have reached completes the proof.

Remark 2.2. Let $y \in H$ and let $x_{\varepsilon}(y)$ denote the unique solution of the regularized optimization problem

$$
\min _{x \in C} f_{\varepsilon}(x):=\frac{1}{2}\|A x-b\|^{2}+\frac{1}{2} \varepsilon\|x-y\|^{2} .
$$


Let $P_{S}$ be the nearest point projection from $H$ onto $S$. Then, we can prove the following results:

(i) $S \neq \varnothing$ if and only if $\left\{x_{\varepsilon}(y)\right\}$ is bounded as $\varepsilon \downarrow 0$. In this case, $\left\{x_{\varepsilon}(y)\right\}$ converges strongly as $\varepsilon \downarrow 0$ to the solution of (1.1) closest to $y$ (i.e., $P_{S} y$ );

(ii) $S=\varnothing$ if and only if $\lim _{\varepsilon} \downarrow 0 \mid x_{\varepsilon}(y) \|=\infty$.

\section{Iterative algorithm}

In this section, we will introduce an iterative algorithm which produces a sequence that converges strongly to the unique solution of the regularized problem (1.2) in the case where the set $C$ of constraints is the intersection of finitely many closed convex subsets of $H$. We need some lemmas. Lemma 3.1 is elementary (see [14] for a proof). Lemma 3.2 is an easy exercise in an inner product space. Lemma 3.3 is well known in the fixed-point theory of nonexpansive mappings (see, e.g., Goebel and Kirk [7]). Lemma 3.4 may be known. A proof is, however, included.

LEMMA 3.1. Let $\left\{s_{n}\right\}$ be a sequence of nonnegative numbers satisfying

$$
s_{n+1} \leq\left(1-\alpha_{n}\right) s_{n}+\alpha_{n} \beta_{n}, \quad n \geq 0
$$

where $\left\{\alpha_{n}\right\}$ and $\left\{\beta_{n}\right\}$ are sequences of real numbers such that

(i) $\left\{\alpha_{n}\right\} \subset[0,1]$ and $\sum_{n=0}^{\infty} \alpha_{n}=\infty$ or equivalently,

$$
\prod_{n=0}^{\infty}\left(1-\alpha_{n}\right):=\lim _{n \rightarrow \infty} \prod_{k=0}^{n}\left(1-\alpha_{k}\right)=0
$$

(ii) $\limsup \operatorname{su}_{n \rightarrow \infty} \beta_{n} \leq 0$, or $\sum_{n} \alpha_{n} \beta_{n}$ is convergent.

Then $\lim _{n \rightarrow \infty} s_{n}=0$.

Lemma 3.2. Let $H$ be a Hilbert space. Then $\|x+y\|^{2} \leq\|x\|^{2}+2\langle y, x+y\rangle$, for all $x, y \in H$.

Lemma 3.3. Let $D$ be a closed convex subset of $H$ and $T: D \rightarrow H$ a nonexpansive mapping with a fixed point. If $\left\{x_{n}\right\}$ is a sequence in $D$ such that $x_{n} \rightarrow x$ weakly and $(I-T) x_{n} \rightarrow y$ strongly, then $(I-T) x=y$. (Here $I$ is the identity operator on $\left.H.\right)$

Lemma 3.4 (the optimality condition for (1.2)). The point $x_{\varepsilon} \in C$ solves (1.2) if and only if

$$
\left\langle x-x_{\varepsilon}, A^{*} b-\left(A^{*} A+\varepsilon I\right) x_{\varepsilon}\right\rangle \leq 0, \quad x \in C,
$$

where $A^{*}$ is the adjoint of $A$. 
Proof. Assume that $x_{\varepsilon} \in C$ is the minimizer of $f_{\varepsilon}$ over $C$. We have, for any $x \in C$,

$$
\begin{aligned}
& 0 \leq \lim _{t \downarrow 0} \frac{1}{t}\left[f_{\varepsilon}\left(x_{\varepsilon}+t\left(x-x_{\varepsilon}\right)\right)-f_{\varepsilon}\left(x_{\varepsilon}\right)\right] \\
&=\lim _{t \downarrow 0}\left[\left\langle A x_{\varepsilon}-b, A\left(x-x_{\varepsilon}\right)\right\rangle+\varepsilon\left\langle x_{\varepsilon}, x-x_{\varepsilon}\right\rangle\right. \\
&\left.\quad+\frac{t}{2}\left(\left\|A\left(x-x_{\varepsilon}\right)\right\|^{2}+\varepsilon\left\|x-x_{\varepsilon}\right\|^{2}\right)\right] \\
&=\left\langle A^{*}\left(A x_{\varepsilon}-b\right)+\varepsilon x_{\varepsilon}, x-x_{\varepsilon}\right\rangle \\
&=\left\langle\left(A^{*} A+\varepsilon I\right) x_{\varepsilon}-A^{*} b, x-x_{\varepsilon}\right\rangle .
\end{aligned}
$$

Assume next that (3.3) holds. Calculations yield the derivative

$$
f^{\prime}(x)=\left(A^{*} A+\varepsilon I\right) x-A^{*} b .
$$

Hence, by the subdifferential inequality, we obtain, for all $x \in C$,

$$
\begin{aligned}
f(x) & \geq f\left(x_{\varepsilon}\right)+\left\langle f^{\prime}\left(x_{\varepsilon}\right), x-x_{\varepsilon}\right\rangle \\
& =f\left(x_{\varepsilon}\right)+\left\langle\left(A^{*} A+\varepsilon I\right) x_{\varepsilon}-A^{*} b, x-x_{\varepsilon}\right\rangle \geq f\left(x_{\varepsilon}\right) .
\end{aligned}
$$

This shows that $x_{\varepsilon}$ is indeed a minimizer of $f_{\varepsilon}$ over $C$.

We now assume that $S \neq \varnothing$ and look for the minimum norm solution of (1.1) in the case where

$$
C=\bigcap_{i=1}^{N} C_{i},
$$

where $N \geq 1$ is an integer and $\left\{C_{1}, \ldots, C_{N}\right\}$ is a finite collection of closed convex subsets of a Hilbert space $H$. We assume that for each $i, C_{i}$ is the fixed-point set of a nonexpansive mapping $T_{i}$ (i.e., $C_{i}=\left\{x \in H: T_{i} x=x\right\}$ ). Recall that a mapping $T: H \rightarrow H$ is nonexpansive if $\|T x-T y\| \leq\|x-y\|$ for all $x, y \in H$. It is well known that the (nearest point) projection $P_{D}$ from $H$ onto a closed convex subset $D$ of $H$ is nonexpansive. Note that each $C_{i}$ is the fixed-point set of the projection $P_{C_{i}}$.

We now introduce the iterative algorithm. Let a sequence $\left\{\alpha_{n}\right\}$ of real numbers in the interval $[0,1]$ be given. For $\varepsilon>0$, we put

$$
B \equiv B_{\varepsilon}:=A^{*} A+\varepsilon I, \quad u:=A^{*} b .
$$


Starting with an arbitrary initial guess $x_{0} \in H$, we define the sequence $\left\{x_{n}\right\}$ recursively by the following algorithm:

$$
\begin{aligned}
x_{1} & =\left(I-\alpha_{1} B\right) T_{1} x_{0}+\alpha_{1} u, \\
x_{2} & =\left(I-\alpha_{2} B\right) T_{2} x_{1}+\alpha_{2} u, \\
& \vdots \\
x_{N} & =\left(I-\alpha_{N} B\right) T_{N} x_{N-1}+\alpha_{N} u, \\
x_{N+1} & =\left(I-\alpha_{N+1} B\right) T_{1} x_{N}+\alpha_{N+1} u,
\end{aligned}
$$

We can rewrite the algorithm in a more compact form

$$
x_{n+1}=\left(I-\alpha_{n+1} B\right) T_{n+1} x_{n}+\alpha_{n+1} u, \quad n \geq 0,
$$

where $T_{n}=T_{n \bmod N}$ and the $\bmod$ function takes values in $\{1,2, \ldots, N\}$.

Remark 3.5. The algorithm is a modification of an iteration scheme proposed by Halpern [8] and developed by Lions [9], Wittmann [13], Reich [10, 11], Bauschke [1], $\mathrm{Xu}[14,15]$, and others. The algorithm and its variants apply to the convex feasibility problem (CFP). (See $[2,4]$ for some of the recent developments regarding the CFP.)

We now prove the main result of the paper. Some related work can also be found in a paper by Deutsch and Yamada [5].

Theorem 3.6. Assume that

(1) $\alpha_{n} \rightarrow 0$;

(2) $\sum_{n} \alpha_{n}=\infty$;

(3) $\alpha_{n} / \alpha_{n+N} \rightarrow 1$ or $\sum_{n}\left|\alpha_{n}-\alpha_{n+N}\right|<\infty$;

(4) $C=\bigcap_{i=1}^{N} F\left(T_{i}\right)=F\left(T_{1} T_{2} \cdots T_{N}\right)=F\left(T_{N} T_{1} \cdots T_{N-1}\right)=\cdots=F\left(T_{2} T_{3}\right.$ $\left.\cdots T_{N} T_{1}\right)$.

Then, the sequence $\left(x_{n}\right)$ generated by algorithm (3.10) converges in norm to the unique solution $x_{\varepsilon}$ of (1.2).

Proof. We will divide the proof into several steps.

(1) First, we show that the sequence $\left\{x_{n}\right\}$ is bounded. Indeed, since $B$ is selfadjoint, we have that for any $0<\alpha<\left(\|A\|^{2}+\varepsilon\right)^{-1}, I-\alpha B$ is positive. Hence,

$$
\|I-\alpha B\|=\sup _{\|x\|=1}\langle(I-\alpha B) x, x\rangle \leq 1-\alpha \varepsilon
$$


(Without loss of generality, we may assume throughout the proof that $\alpha_{n}<$ $\left(\|A\|^{2}+\varepsilon\right)^{-1}$ for all $n$.) It follows from (3.11) that for $p \in C$,

$$
\begin{aligned}
\left\|x_{n+1}-p\right\| & =\left\|\left(I-\alpha_{n+1} B\right)\left(T_{n+1} x_{n}-p\right)+\alpha_{n+1}(u-B p)\right\| \\
& \leq\left(1-\varepsilon \alpha_{n+1}\right)\left\|x_{n}-p\right\|+\alpha_{n+1}\|u-B p\| .
\end{aligned}
$$

Hence, by induction, we obtain

$$
\left\|x_{n}-p\right\| \leq \max \left\{\left\|x_{0}-p\right\|, \frac{\|u-B p\|}{\varepsilon}\right\}, \quad n \geq 0 .
$$

(2) Next, we note that $\left\|x_{n+1}-T_{n+1} x_{n}\right\| \rightarrow 0$. This follows from assumption (1), step (1), and the fact that $\left\|x_{n+1}-T_{n+1} x_{n}\right\|=\alpha_{n+1}\left\|u-B T_{n+1} x_{n}\right\|$.

(3) Now, we prove that $\left\|x_{n+N}-x_{n}\right\| \rightarrow 0$. Indeed, we have (note that $T_{n+N}=$ $\left.T_{n}\right)$

$$
\begin{aligned}
\left\|x_{n+N}-x_{n}\right\|= & \|\left(I-\alpha_{n+N} B\right)\left(T_{n+N} x_{n+N-1}-T_{n} x_{n-1}\right) \\
& +\left(\alpha_{n+N}-\alpha_{n}\right)\left(u-B T_{n} x_{n-1}\right) \| \\
\leq & \left(1-\varepsilon \alpha_{n+N}\right)|| x_{n+N-1}-x_{n-1} \|+M\left|\alpha_{n+N}-\alpha_{n}\right| \\
= & \left(1-\varepsilon \alpha_{n+N}\right)|| x_{n+N-1}-x_{n-1} \|+\varepsilon \alpha_{n+N} \beta_{n},
\end{aligned}
$$

where $M$ is a constant such that $\left\|u-B T_{n} x_{n-1}\right\| \leq M$ for all $n$ and $\beta_{n}:=$ $M \varepsilon^{-1} \alpha_{n+N}^{-1}\left|\alpha_{n+N}-\alpha_{n}\right|$. Applying Lemma 3.1 together with assumptions (2) and (3), we obtain that $\left\|x_{n+N}-x_{n}\right\| \rightarrow 0$.

(4) Next, we observe that $x_{n}-T_{n+N} \cdots T_{n+1} x_{n} \stackrel{s}{\rightarrow} 0$.

Indeed, noting that each $T_{n}$ is nonexpansive and using step (2), we observe that

$$
\begin{array}{r}
x_{n+N}-T_{n+N} x_{n+N-1} \stackrel{s}{\longrightarrow} 0, \\
T_{n+N} x_{n+N-1}-T_{n+N} T_{n+N-1} x_{n+N-2} \stackrel{s}{\longrightarrow} 0, \\
\vdots \\
T_{n+N} \cdots T_{n+2} x_{n+1}-T_{n+N} \cdots T_{n+1} x_{n} \stackrel{s}{\longrightarrow} 0 .
\end{array}
$$

Adding up and using step (3), we see that $x_{n}-T_{n+N} \cdots T_{n+1} x_{n} \stackrel{s}{\rightarrow} 0$.

(5) Now, we show that $\limsup _{n \rightarrow \infty}\left\langle u-B x_{\varepsilon}, x_{n}-x_{\varepsilon}\right\rangle \leq 0$, where $x_{\varepsilon}$ is the unique solution of (1.2). 
Take a subsequence $\left\{x_{n_{j}}\right\}$ of $\left\{x_{n}\right\}$ such that

$$
\limsup _{n \rightarrow \infty}\left\langle u-B x_{\varepsilon}, x_{n}-x_{\varepsilon}\right\rangle=\lim _{j \rightarrow \infty}\left\langle u-B x_{\varepsilon}, x_{n_{j}}-x_{\varepsilon}\right\rangle .
$$

Since $\left\{x_{n}\right\}$ is bounded, we may also assume that there exists some $\tilde{x} \in H$ such that $x_{n_{j}} \stackrel{w}{\rightarrow} \tilde{x}$. Since the pool of mappings is finite, we may further assume (passing to a further subsequence if necessary) that, for some $i \in\{1,2, \ldots, N\}$,

$$
T_{n_{j}} \equiv T_{i}, \quad \forall j \geq 1
$$

It follows from step (4) that

$$
x_{n_{j}}-T_{i+N} \cdots T_{i+1} x_{n_{j}} \stackrel{s}{\rightarrow} 0 .
$$

Lemma 3.3 then ensures that the weak limit $\tilde{x}$ of $\left\{x_{n_{j}}\right\}$ is a fixed point of the mapping $T_{i+N} \cdots T_{i+1}$. This together with assumption (4) implies that $\tilde{x} \in F\left(T_{i+N}\right.$ $\left.\cdots T_{i+1}\right)=C$. Therefore, we have, by (3.16) and Lemma 3.4, that $\limsup _{n \rightarrow \infty}\langle u-$ $\left.B x_{\varepsilon}, x_{n}-x_{\varepsilon}\right\rangle=\left\langle u-B x_{\varepsilon}, \tilde{x}-x_{\varepsilon}\right\rangle \leq 0$.

(6) Finally, we claim that $x_{n} \stackrel{s}{\rightarrow} x_{\varepsilon}$. Indeed, we can write

$$
x_{n+1}-x_{\varepsilon}=\left(I-\alpha_{n+1} B\right)\left(T_{n+1} x_{n}-x_{\varepsilon}\right)+\alpha_{n+1}\left(u-B x_{\varepsilon}\right) .
$$

Apply Lemma 3.2 to get

$$
\begin{aligned}
\left\|x_{n+1}-x_{\varepsilon}\right\|^{2} & \leq\left\|\left(I-\alpha_{n+1} B\right)\left(T_{n+1} x_{n}-x_{\varepsilon}\right)\right\|^{2}+2 \alpha_{n+1}\left\langle u-B x_{\varepsilon}, x_{n+1}-x_{\varepsilon}\right\rangle \\
& \leq\left(1-\varepsilon \alpha_{n+1}\right)\left\|x_{n}-x_{\varepsilon}\right\|^{2}+2 \alpha_{n+1}\left\langle u-B x_{\varepsilon}, x_{n+1}-x_{\varepsilon}\right\rangle .
\end{aligned}
$$

Using Lemma 3.1 and step (5), we now conclude that $\left\|x_{n}-x_{\varepsilon}\right\|^{2} \rightarrow 0$.

Remark 3.7. Assumption (4) in Theorem 3.6 is automatically satisfied if each $T_{i}$ is firmly nonexpansive (i.e., $T_{i}$ satisfies the property: $\left\langle x-y, T_{i} x-T_{i} y\right\rangle \geq \| T_{i} x-$ $T_{i} y \|^{2}$, for $\left.x, y \in H\right)$. Since a projection is firmly nonexpansive, we have the following consequence of Theorem 3.6.

Corollary 3.8. Assume that

(1) $\alpha_{n} \rightarrow 0$;

(2) $\sum_{n} \alpha_{n}=\infty$;

(3) $\alpha_{n} / \alpha_{n+N} \rightarrow 1$ or $\sum_{n}\left|\alpha_{n}-\alpha_{n+N}\right|<\infty$.

Let $x_{0} \in H$ be chosen arbitrarily and let $\left\{x_{n}\right\}$ be generated by the iterative algorithm

$$
x_{n+1}=\left(I-\alpha_{n+1} B\right) P_{n+1} x_{n}+\alpha_{n+1} u, \quad n \geq 0,
$$

where $P_{k}=P_{C_{k}}(1 \leq k \leq N)$. Then, $\left\{x_{n}\right\}$ converges in norm to the unique solution $x_{\varepsilon}$ of (1.2). 
Remark 3.9. We have used two steps to find a solution of problem (1.1); that is, we first find the unique solution $x_{\varepsilon}$ of the regularized problem (1.2) and then approximate $x_{\varepsilon}$ via the iterative algorithm (3.10). It is not clear if one can combine the two steps into one. In other words, if one defines a sequence $\left\{x_{n}\right\}$ by

$$
x_{n+1}=\left(I-\alpha_{n+1} B_{\varepsilon_{n}}\right) T_{n+1} x_{n}+\alpha_{n+1} u, \quad n \geq 0,
$$

where $\left\{\varepsilon_{n}\right\} \subset(0,1)$ and $\varepsilon_{n} \rightarrow 0$ as $n \rightarrow \infty$, does the sequence $\left\{x_{n}\right\}$ defined by (3.22) converge to the minimum norm solution of problem (1.1) (if the solution set $S$ of (1.1) is nonempty)?

\section{Acknowledgment}

The first author was partially supported by the Israel Science Foundation founded by the Israel Academy of Sciences and Humanities (Grant 592/00), by the Fund for the Promotion of Research at the Technion, and by the Technion VPR Fund.

\section{References}

[1] H. H. Bauschke, The approximation of fixed points of compositions of nonexpansive mappings in Hilbert space, J. Math. Anal. Appl. 202 (1996), no. 1, 150-159.

[2] H. H. Bauschke and J. M. Borwein, On projection algorithms for solving convex feasibility problems, SIAM Rev. 38 (1996), no. 3, 367-426.

[3] A. Björck, Numerical Methods for Least Squares Problems, Society for Industrial and Applied Mathematics (SIAM), Pennsylvania, 1996.

[4] P. L. Combettes, Hilbertian convex feasibility problem: convergence of projection methods, Appl. Math. Optim. 35 (1997), no. 3, 311-330.

[5] F. Deutsch and I. Yamada, Minimizing certain convex functions over the intersection of the fixed point sets of nonexpansive mappings, Numer. Funct. Anal. Optim. 19 (1998), no. 1-2, 33-56.

[6] H. W. Engl, M. Hanke, and A. Neubauer, Regularization of Inverse Problems, Mathematics and its Applications, vol. 375, Kluwer Academic Publishers, Dordrecht, 1996.

[7] K. Goebel and W. A. Kirk, Topics in Metric Fixed Point Theory, Cambridge Studies in Advanced Mathematics, vol. 28, Cambridge University Press, Cambridge, 1990.

[8] B. Halpern, Fixed points of nonexpanding maps, Bull. Amer. Math. Soc. 73 (1967), 957-961.

[9] P.-L. Lions, Approximation de points fixes de contractions, C. R. Acad. Sci. Paris Sér. A-B 284 (1977), no. 21, A1357-A1359 (French).

[10] S. Reich, Strong convergence theorems for resolvents of accretive operators in Banach spaces, J. Math. Anal. Appl. 75 (1980), no. 1, 287-292.

[11] _ Approximating fixed points of nonexpansive mappings, Panamer. Math. J. 4 (1994), no. 2, 23-28.

[12] A. N. Tikhonov and V. Y. Arsenin, Solutions of Ill-posed Problems, Wiley, New York, 1997.

[13] R. Wittmann, Approximation of fixed points of nonexpansive mappings, Arch. Math. (Basel) 58 (1992), no. 5, 486-491. 


\section{Constrained least squares problem}

[14] H. K. Xu, Another control condition in an iterative method for nonexpansive mappings, Bull. Austral. Math. Soc. 65 (2002), no. 1, 109-113.

[15] _ An iterative approach to quadratic optimization, J. Optim. Theory Appl. 116 (2003), no. 3, 659-678.

Simeon Reich: Department of Mathematics, The Technion - Israel Institute of Technology, 32000 Haifa, Israel

E-mail address: sreich@techunix.technion.ac.il

Hong-Kun Xu: Department of Mathematics, University of Durban-Westville, Private Bag X54001, Durban 4000, South Africa

E-mail address: hkxu@pixie.udw.ac.za 


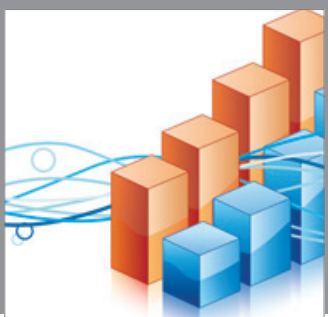

Advances in

Operations Research

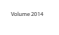

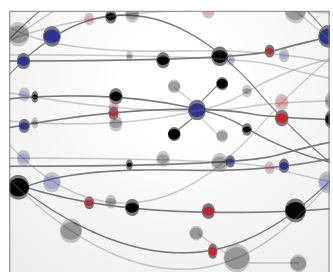

\section{The Scientific} World Journal
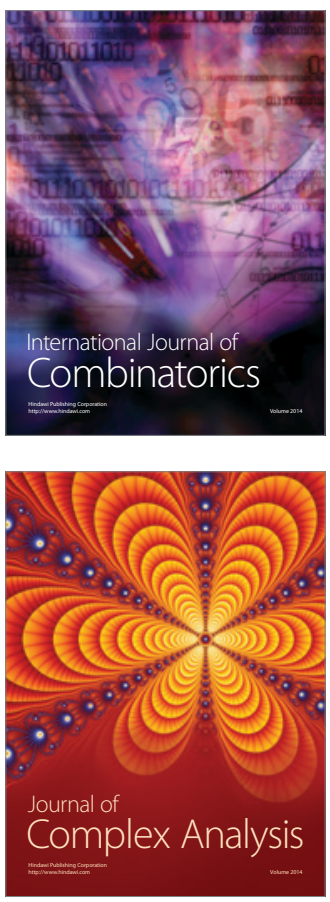

International Journal of

Mathematics and

Mathematical

Sciences
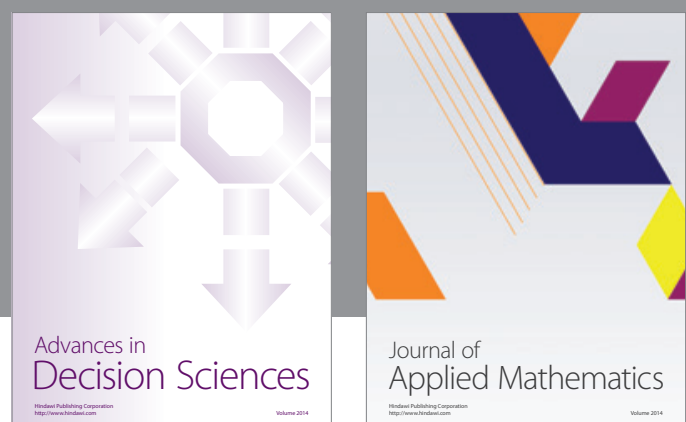

Journal of

Applied Mathematics
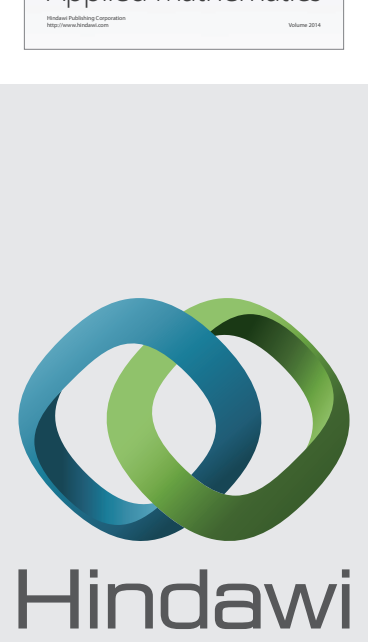

Submit your manuscripts at http://www.hindawi.com
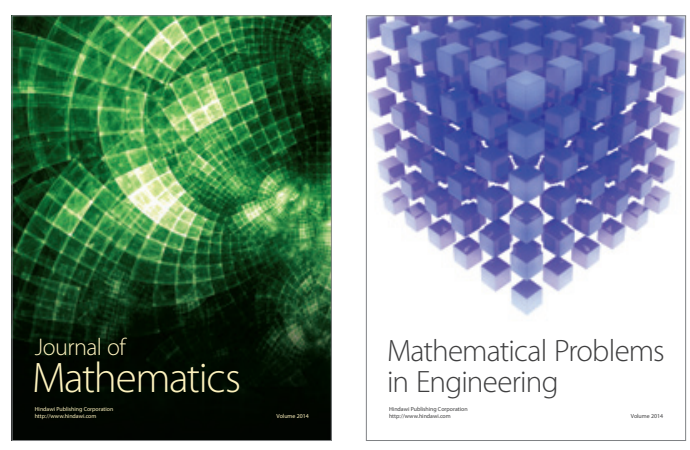

Mathematical Problems in Engineering
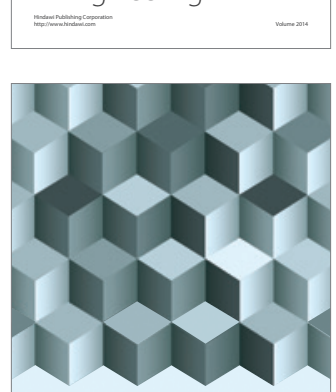

Journal of

Function Spaces
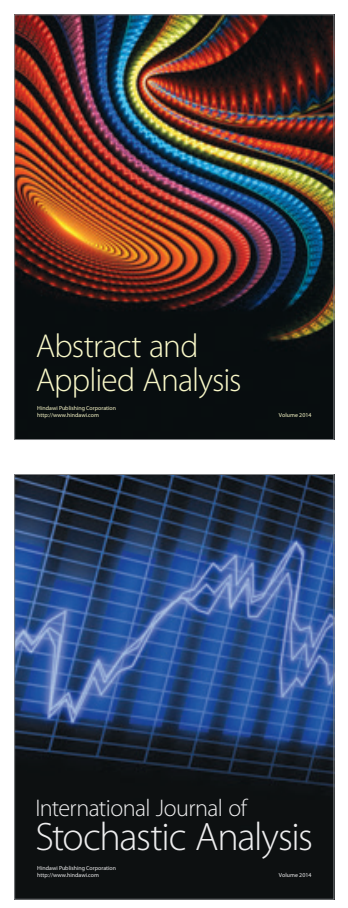

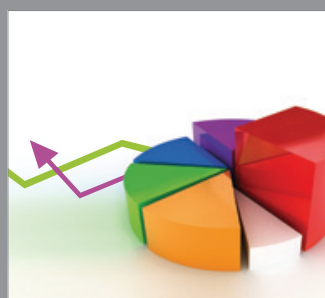

ournal of

Probability and Statistics

Promensencen
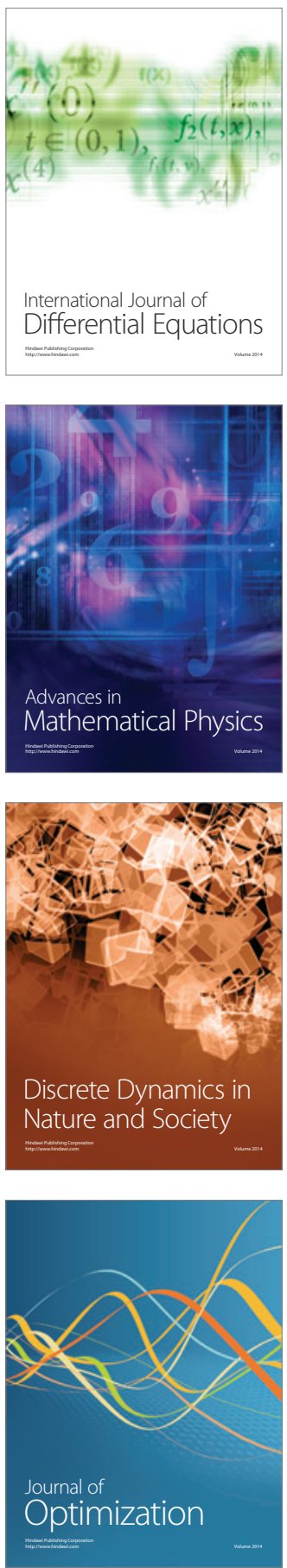